\title{
Accurate Measurement of Isotope Amount Ratios of Lead in Bronze with Multicollector Inductively Coupled Plasma Mass Spectrometry
}

\author{
Kyoung-Seok Lee ${ }^{1, *}$, Jin-il Kim ${ }^{1,2}$, Yong-Hyeon Yim ${ }^{1}$, Euijin Hwang ${ }^{1}$, and Tae Kyu Kim ${ }^{2}$ \\ ${ }^{I}$ Korea Research Institute of Standards and Science (KRISS),Daejeon 305-340, Korea \\ ${ }^{2}$ Department of Chemistry, Pusan National University, Busan 609-735, Korea
}

Received December 13, 2013; Accepted December 19, 2013

First published on the web December 30, 2013; DOI: 10.5478/MSL.2013.4.4.87

\begin{abstract}
Isotope amount ratios of lead in a bronze sample have been successfully determined using multicollector inductively coupled plasma mass spectrometry (MC-ICP-MS). Matrix separation conditions were tested and optimized using ion exchange chromatography with anion-exchange resin, AG1-X8, and sequential elution of the $0.5 \mathrm{M} \mathrm{HBr}$ and $7 \mathrm{M} \mathrm{HNO}_{3}$ to separate lead from very high contents of copper and tin in bronze matrix. Mercury was also removed efficiently in the optimized separation condition. The instrumental isotope fractionation of lead in the MC-ICP-MS measurement was corrected by the external standard sample bracketing method using an external standard, NIST SRM 981 lead common isotope ratio standard followed by correction of procedure blank to obtain reliable isotope ratios of lead. The isotope ratios, ${ }^{206} \mathrm{~Pb} /{ }^{204} \mathrm{~Pb},{ }^{207} \mathrm{~Pb} /{ }^{204} \mathrm{~Pb},{ }^{208} \mathrm{~Pb} /{ }^{204} \mathrm{~Pb}$, and ${ }^{208} \mathrm{~Pb} /{ }^{206} \mathrm{~Pb}$, of lead were determined as $18.0802 \pm 0.0114,15.5799 \pm 0.0099,38.0853 \pm 0.0241$, and $2.1065 \pm 0.0004$, respectively, and the determined isotope ratios showed good agreement with the reference values of an international comparison for the same sample within the stated uncertainties
\end{abstract}

Key words: isotope ratio, lead, MC-ICP-MS, bronze, matrix separation

\section{Introduction}

Lead $(\mathrm{Pb})$ has four stable isotopes, ${ }^{204} \mathrm{~Pb},{ }^{206} \mathrm{~Pb},{ }^{207} \mathrm{~Pb},{ }^{208} \mathrm{~Pb}$ with natural abundances of $1.4 \%, 24.1 \%, 22.1 \%$, and $52.4 \%$, respectively. ${ }^{1}$ Since the three isotopes of $\mathrm{Pb},{ }^{206} \mathrm{~Pb}$, ${ }^{207} \mathrm{~Pb}$, and ${ }^{208} \mathrm{~Pb}$ are radiogenic end products of the radioactive decay chains of uranium series $\left({ }^{238} \mathrm{U} \rightarrow{ }^{206} \mathrm{~Pb}\right)$, actinium series $\left({ }^{235} \mathrm{U} \rightarrow{ }^{207} \mathrm{~Pb}\right)$, and thorium series $\left({ }^{232} \mathrm{Th} \rightarrow{ }^{208} \mathrm{~Pb}\right)$, the isotopic composition of lead are expected to change in their relative abundances depending on the geological environments, transport processes, or human nuclear activities. This unique characteristic of $\mathrm{Pb}$ allows the use of $\mathrm{Pb}$ isotope ratios as an important tracer in many useful applications including the study of natural isotope variations, geology, archeology, and investigations on the provenance of specific samples as well as the information on the source of atmospheric pollution. ${ }^{2-5}$ However, the variation of isotopic

\section{Open Access}

*Reprint requests to Dr. Kyoung-Seok Lee

E-mail: kslee@kriss.re.kr

All MS Letters content is Open Access, meaning it is accessible online to everyone, without fee and authors' permission. All MS Letters content is published and distributed under the terms of the Creative Commons Attribution License (http://creativecommons.org /licenses/by/3.0/). Under this license, authors reserve the copyright for their content; however, they permit anyone to unrestrictedly use, distribute, and reproduce the content in any medium as far as the original authors and source are cited. For any reuse, redistribution, or reproduction of a work, users must clarify the license terms under which the work was produced. compositions of lead depending on their sources necessitates measurements of individual isotope ratios of the sample and calibration standard for accurate determination of amount contents of lead in a sample using isotope dilution mass spectrometry (IDMS), which has been widely accepted as a definitive reference method of analysis. ${ }^{6}$ Accurate determination of $\mathrm{Pb}$ molar mass also relies on the measurement of isotopic compositions.

Many applications of isotope amount ratio measurement require determination of quite small variations and, commonly used quadrupole inductively coupled plasma mass spectrometers (ICP-MS) do not provide enough precision required to distinguish very small differences in the ratios. Therefore, specially designed instruments such as thermal ionization mass spectrometry (TIMS) and multicollectorinductively coupled plasma mass spectrometry (MC-ICP-MS) have been increasingly used for the determination of isotope amount ratios of lead. These instruments use magnetic sector to separate ion beams with different mass-to-charge ratios and collect them into the series of Faraday cup detectors for monitoring ion currents of different isotopes simultaneously. ${ }^{2}$ TIMS has been used predominantly for the accurate isotope ratio determinatione before the development of MC-ICP-MS and good repeatability can be achieved because thermal ionization produces ions with a very small kinetic energy dispersion of less than $1 \mathrm{eV}$. Time-consuming sample preparation including sample pretreatment and sample loading on a filament is required in TIMS, however, to correct for 
mass-dependent instrumental fractionation (also referred to as mass bias) due to preference of evaporation of the light isotope during thermal ionization. After MC-ICP-MS has been introduced, it is becoming more and more popular instrument for isotope ratio analysis of many elements taking advantage of high ionization efficiency of argon plasma for most elements in periodic table and relatively short measurement time required to get reliable results. ${ }^{7}$

In the isotope ratio analysis of lead, the correction of the instrumental isotope fractionation is crucial to obtain accurate and reliable isotope ratios. Isotopic fractionation in MC-ICP-MS is mostly due to space charge effects, which make the heavier isotope ions transported more efficiently at the interface between ICP source and MS and detected more efficiently. In MC-ICP-MS measurements, ions produced by high temperature plasma have higher kinetic energy spread compared with ions generated by thermal ionization and fractionations of different isotopes are severer. Therefore, the measured isotope ratios of lead should be corrected for this fractionations using appropriate method. ${ }^{8}$

In this study, multicollector inductively coupled plasma mass spectrometry (MC-ICP-MS) was used to determine the isotope amount ratios of lead in a bronze sample. Since the bronze sample contains very large amount of copper and tin compared with lead present in a trace level, matrix separation using anion-exchange resin was optimized and applied. In order to correct for the instrumental isotope fractionation, standard sample bracketing method ${ }^{2,8}$ was also applied using an external isotope ratio standard. Then, the determined isotope ratios of lead were compared with the KCRV (key comparison reference value) which is a consensus value calculated from reported results of national metrology institutes participating for an international comparison of CCQM (Consultative Committee for Amount of Substance) IAWG (Inorganic Analysis Working group).

\section{Experimental}

\section{Materials and reagents.}

The bronze swarf, for CCQM-K98 was provided by the BAM (Federal Institute for Materials Research and Testing) in Germany, which is the pilot laboratory for the Key Comparison study. The SRM 981, common lead isotopic standard, was purchased from the National Institute of Standards and Technology (NIST, USA) and dissolved in 5\% nitric acid as a stock solution. Nitric acids with various concentrations used throughout this study were prepared just before sample preparation by diluting the concentrated nitric acid $(\geq 65 \%)$ with deionized water (18 MW $\Omega \cdot \mathrm{cm}$ resistivity) from Milli-Q RG purification system (Millipore, USA). The concentrated nitric acid was obtained by sub-boiling distillation of commercially available concentrated nitric acid from DONGWOO FINE-CHEM (Iksan, Korea). Hydrobromic acid was purchased from Sigma-Aldrich
(Milwaukee, Wl, USA) and used without further purification. The AG1-X8 resin (100-200 mesh) was obtained from BioRad Laboratories, Inc. (Hercules, CA, USA).

\section{Sample preparation and matrix separation.}

One hundred milligram chips of bronze swarf were completely dissolved in $30 \mathrm{~mL}$ of $3 \mathrm{M}$ nitric acid and then the sample solution was dried at $95^{\circ} \mathrm{C}$ using heating block. The residue was reconstituted with $2 \mathrm{~mL}$ of $0.5 \mathrm{M} \mathrm{HBr}$ and loaded onto the preconditioned AG1-X8 resin for lead separation from the bronze matrix. Five milliliter volume of column was packed with $1.5 \mathrm{~mL}$ of AG1-X8 resin. The resin was cleaned by elution of $30 \mathrm{~mL} 7 \mathrm{M} \mathrm{HNO}_{3}$ and followed by $60 \mathrm{~mL} 0.5 \mathrm{M} \mathrm{HBr}$. Then, the sample in $0.5 \mathrm{M} \mathrm{HBr}$ was loaded onto the pre-conditioned resin. Lead was retained on the resin but the major constituents, copper and tin, were eluted by sequential addition of $10 \mathrm{~mL} 0.5 \mathrm{M} \mathrm{HBr}$. After the blue color of copper ions was removed in the column, the lead retained on the resin was eluted by sequential $10 \mathrm{~mL}$ of $7 \mathrm{M} \mathrm{HNO}_{3}$. Every fractions from individual elution steps are collected and their contents of copper, tin, and lead were monitored by Element 2 ICP-MS (Thermo Fisher Scientific Inc., Bremen, Germany). Eluted lead solution in $7 \mathrm{M} \mathrm{HNO}_{3}$ was evaporated to dryness and reconstituted by $5 \% \mathrm{HNO}_{3}$ for matrix matching before isotope ratio measurement. The final test solution was diluted to approximately $10 \mathrm{mg} / \mathrm{kg}$ considering the dynamic range of the Faraday detectors of MC-ICP-MS instrument. Procedure blanks were also prepared and went through the same procedures of sample preparation and matrix separation with the samples.

\section{Lead isotope ratio measurements.}

All isotope ratio measurements were performed using a Neptune (Thermo Fisher Scientific Inc., Bremen, Germany) multicollector inductively coupled plasma mass spectrometer (MC-ICP-MS). The Neptune MC-ICP-MS is a double-

Table 1. Optimized operating conditions and data acquisition parameters of MC-ICP-MS for isotope ratio measurements of lead.

\begin{tabular}{cc}
\hline \hline Sampler/skimmer cones & $\mathrm{Ni}$ \\
\hline Plasma gas flow & $15 \mathrm{~L} / \mathrm{min}$ \\
Auxiliary gas flow & $0.7 \mathrm{~L} / \mathrm{min}$ \\
Nebulizer gas flow & $1.335 \mathrm{~L} / \mathrm{min}$ \\
plasma RF power & $1200 \mathrm{~W}$ \\
Resolution & Low resolution $(\mathrm{R} \sim 300)$ \\
Integration time & 4.194 seconds \\
Idle time & 3 seconds \\
No. of spectra acquired & 9 blocks' $\times 3$ cycles $/$ block \\
Acquisition & Static mode \\
Cup configuration & $\mathrm{L} 3::^{202} \mathrm{Hg}, \mathrm{L} 2::^{203} \mathrm{~T} 1, \mathrm{~L} 1:{ }^{204} \mathrm{~Pb}, \mathrm{~Pb},:^{207} \mathrm{~Pb}$, \\
$\mathrm{H} 3:{ }^{208} \mathrm{~Pb}$ \\
\hline
\end{tabular}


focusing magnetic sector type instrument of forward NierJohnson geometry and has 9 Faraday collectors and 1 secondary electron multiplier. Further details of the instrument design can be found elsewhere. ${ }^{9}$ The sample solutions were self-aspirated into the quartz dual spray chamber by PFA micro-concentric nebulizer and sample droplets were introduced high temperature plasma through the platinum injector and quartz torch. The operating conditions of MCICP-MS and data acquisition parameters are summarized in Table 1. The raw data obtained were first corrected for the procedural blank. Then, the correction for mass dependent isotope fractionation of lead was performed by standard sample bracketing technique using NIST SRM 981 common lead isotopic standard. In this correction technique, back to back measurements of SRM 981 standard were done before and after a measurement of sample, and the isotope ratios of a sample measurement were corrected by the isotope fractionation factors which were obtained from the certified isotope ratios and the measured isotope ratios of SRM 981.

\section{Results and Discussion}

\section{Matrix separation.}

Since the major constituents of the bronze were copper $(\sim 94 \%)$ and tin $(\sim 6 \%)$ which can cause an isotope fractionation of lead by matrix effect, it is necessary to separate lead from the bronze matrix. In this study, anion-exchange chromatography using AG1-X8 resin with hydrobromic acid and nitric acid was applied..$^{10}$ Before the analysis of sample, a standard mixture solution having similar elemental composition of copper, tin, and lead with the bronze sample was used to optimize experimental conditions for the matrix separation of lead. Figure 1(a) and 1(b) show the intensities of
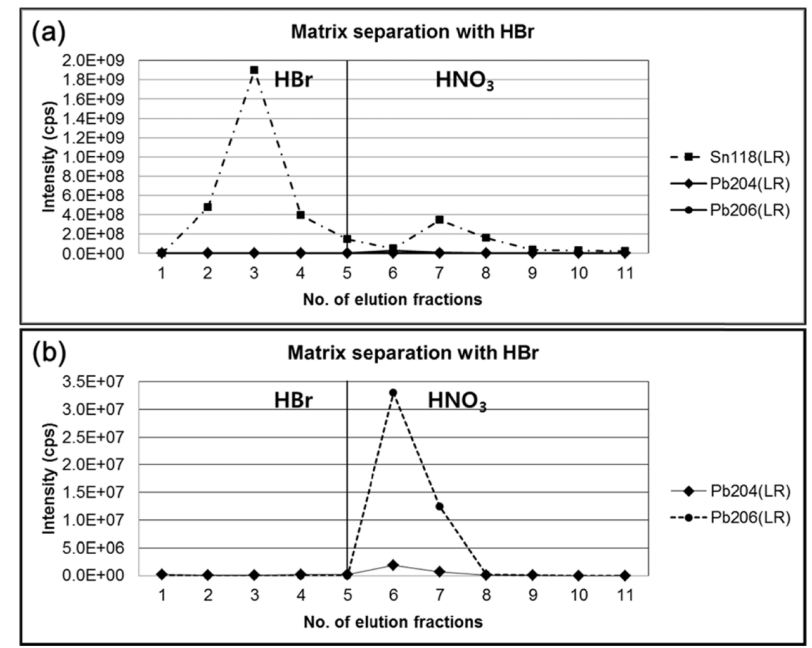

Figure 1. Elution profiles of tin and lead with $0.5 \mathrm{M} \mathrm{HBr}$ washing (fractions of 1-5) followed by $7 \mathrm{M} \mathrm{HNO}_{3}$ elution (fractions of 6-11): (a) with Sn ion intensities, (b) with only lead intensities. tin and lead in each elution fraction obtained by ICP-MS with single secondary electron amplifier. When the standard mixture with blue color of excessive copper ions dissolved in $0.5 \mathrm{M} \mathrm{HBr}$ was loaded on the pre-conditioned resin, copper was easily removed by $0.5 \mathrm{M} \mathrm{HBr}$. During elution with $\mathrm{HBr}$, lead was retained strongly on the resin as bromide complex anion of lead. Then, lead was eluted from the resin by $7 \mathrm{M}$ $\mathrm{HNO}_{3}$. Finally, the collected solutions were evaporated to dryness and reconstituted with $5 \% \mathrm{HNO}_{3}$ for isotope ratio measurements using MC-ICP-MS.

However, it is still challenging to separate trace level of lead from large amounts of tin where the amount ratio of tin and lead is four orders of magnitude because trace level or minor fraction of tin still retains in the resin as weak bromide complex. ${ }^{11}$ After washing with $90 \mathrm{~mL}$ of $0.5 \mathrm{M}$ $\mathrm{HBr}$, which corresponds to 60 column volumes of anionexchange resin, comparable amount of tin was still observed in the nitric acid elution fraction with lead, although the major fraction of tin has been removed. Presence of residual tin and copper at this level, fortunately, do not make isobaric interferences with isotopes of lead including polyatomic ions in the ICP-MS measurements. Removal of major matrix component in the sample solution is expected to minimize non-spectral interferences in the nebulization and ionization efficiencies, and differential isotopic fractionations of lead between the sample and the standard during isotope ratio measurements are not likely.

In addition, the optimized matrix separation condition was also tested for mercury because ${ }^{204} \mathrm{Hg}^{+}$ion generated from mercury in the sample may cause isobaric interference with ${ }^{204} \mathrm{~Pb}^{+}$and results in bias to the measured isotope amount ratios of lead. Another standard mixture solution with mercury was prepared and loaded on the preconditioned anion exchange resin followed by elution with $0.5 \mathrm{M} \mathrm{HBr}$. Figure 2 shows the ion intensities of mercury and lead in each eluted fractions of $0.5 \mathrm{M} \mathrm{HBr}$, where ${ }^{204} \mathrm{~Pb}$ represents the sum of ion intensities of both ${ }^{204} \mathrm{~Pb}$ and ${ }^{204} \mathrm{Hg}$. Considering the negligible signal from ${ }^{206} \mathrm{~Pb}$ observed at $m / z 206$, which is about 17 times more abundant isotope of lead than ${ }^{204} \mathrm{~Pb}, \mathrm{~m} / \mathrm{z} 204$ ions in Figure 2 are mostly from ${ }^{204} \mathrm{Hg}$. Using the same $0.5 \mathrm{M} \mathrm{HBr}$ washing, rapid removal of ${ }^{200} \mathrm{Hg}$ and ${ }^{204} \mathrm{Hg}$ was observed.

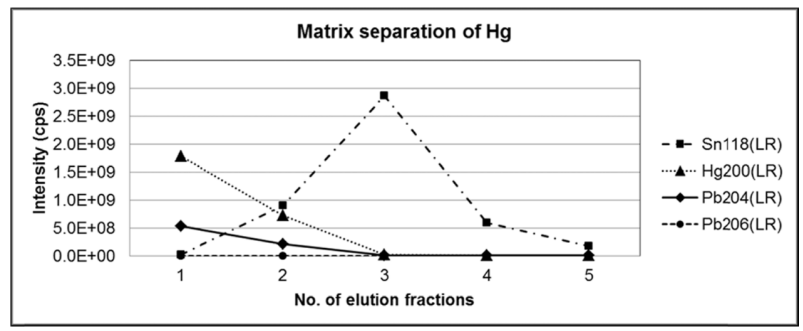

Figure 2. Elution profiles of tin and mercury with $0.5 \mathrm{M} \mathrm{HBr}$ washing (fractions of 1-5) of $\mathrm{Hg}$. The ion intensity of ${ }^{204} \mathrm{~Pb}$ represents the sum of ion intensities of both ${ }^{204} \mathrm{~Pb}$ and ${ }^{204} \mathrm{Hg}$. 
Kyoung-Seok Lee, Jin-il Kim, Yong-Hyeon Yim, Euijin Hwang, and Tae Kyu Kim

Table 2. Determined isotope amount ratios of lead in bronze sample and KCRV isotope amount ratios for the same bronze sample. Isotope amount ratios of SRM 981 are calculated from the atom percent of each isotope given in Certificate of NIST SRM 981.

\begin{tabular}{ccccc}
\hline \hline & $\mathrm{n}\left({ }^{206} \mathrm{~Pb}\right) / \mathrm{n}\left({ }^{204} \mathrm{~Pb}\right)$ & $\mathrm{n}\left({ }^{207} \mathrm{~Pb}\right) / \mathrm{n}\left({ }^{204} \mathrm{~Pb}\right)$ & $\mathrm{n}\left({ }^{208} \mathrm{~Pb}\right) / \mathrm{n}\left({ }^{204} \mathrm{~Pb}\right)$ & $\mathrm{n}\left({ }^{208} \mathrm{~Pb}\right) / \mathrm{n}\left({ }^{206} \mathrm{~Pb}\right)$ \\
\hline Bronze sample & $18.0802 \pm 0.0114$ & $15.5799 \pm 0.0099$ & $38.0853 \pm 0.0241$ & $2.1065 \pm 0.0004$ \\
KCRV & $18.0723 \pm 0.0055$ & $15.5776 \pm 0.0049$ & $38.0751 \pm 0.016$ & $2.10685 \pm 0.00034$ \\
SRM 981 & $16.937 \pm 0.015$ & $15.492 \pm 0.013$ & $36.722 \pm 0.031$ & 2.168 \\
\hline
\end{tabular}

The established matrix separation method was used to prepare the bronze sample solution for MC-ICP-MS measurements. In the MC-ICP-MS measurement of sample, bronze, ${ }^{202} \mathrm{Hg}$ was also measured, in parallel, using one of Faraday collectors, L3, to guarantee no isobaric interference from residual $\mathrm{Hg}$. It turned out that no correction due to the mercury was required.

\section{Isotope ratios of lead.}

Isotope ratios of lead, ${ }^{206} \mathrm{~Pb} /{ }^{204} \mathrm{~Pb},{ }^{207} \mathrm{~Pb} /{ }^{204} \mathrm{~Pb},{ }^{208} \mathrm{~Pb} /{ }^{204} \mathrm{~Pb}$, and ${ }^{208} \mathrm{~Pb} /{ }^{206} \mathrm{~Pb}$, in bronze sample were determined and listed in the Table 2. The instrumental isotope fractionations of the measured isotope ratios were corrected by the bracketing calibration using external standard after correction of the procedure blank. The uncertainties of determined value were calculated following the ISO Guide to the Expression of Uncertainty in Measurement (GUM) guidelines ${ }^{12}$ and expressed as the combined expanded uncertainty with $k=2$ (95\% confidence level). The deviations of isotope ratio measurements for samples, SRM 981 isotope ratio standards, and blank with the uncertainty of the certified isotope ratios of SRM 981 standard used for correction of instrumental isotope fractionation were included for calculation of combined standard uncertainties. The major contribution of standard uncertainty was the uncertainty of the certified values of SRM 981.

The determined results show good agreement with the KCRV of the CCQM comparison even though expanded uncertainties of the determined results were slightly higher than the uncertainties of KCRV. The isotope ratios of lead in bronze sample, ${ }^{206} \mathrm{~Pb} /{ }^{204} \mathrm{~Pb},{ }^{207} \mathrm{~Pb} /{ }^{204} \mathrm{~Pb},{ }^{208} \mathrm{~Pb} /{ }^{204} \mathrm{~Pb}$, are within the natural range of the isotope ratios as tabulated by IUPAC of $17.2 \pm 1.2,15.8 \pm 1.1$, and $37.4 \pm 2.7$, respectively, but ${ }^{208} \mathrm{~Pb} /{ }^{206} \mathrm{~Pb}$ is lower than IUPAC isotope ratio of $2.17 \pm 0.01$.

\section{Conclusion}

Isotope amount ratios of lead in a bronze sample have been successfully determined. For robust isotope ratio measurement for lead from bronze sample matrix with very high contents of copper and tin, matrix separation conditions were tested and optimized using ion exchange chromatography with anion-exchange resin, AG1-X8, and sequential elution of the $0.5 \mathrm{M} \mathrm{HBr}$ and $7 \mathrm{M} \mathrm{HNO}_{3}$. Mercury was also removed efficiently in this separation condition. Correction for instrumental isotope fractionation of lead in MC-ICP-MS was essential to obtain reliable isotope ratios and external standard sample bracketing technique was applied using the NIST SRM 981, lead common isotope ratio standard. The determined isotope ratios of lead show good agreement with the value obtained from international comparison for the same samples indicating comparable measurement capability of KRISS for isotope ratio measurement of lead in metal alloy.

\section{Acknowledgments.}

The authors would like to thank BAM and CCQM IAWG for providing samples. This work was supported by the Korea Research Institute of Standards and Science (KRISS) under the project "Establishing Measurement Standards for Inorganic Analysis", grant 13011022.

\section{References}

1. Cohen, E. R.; Cvitas, T.; Frey, J. G.; Holmström, B.; Kuchitsu, K.; Marquardt, R.; Mills, I.; Pavese, F.; Quack, M.; Stohner, J.; Strauss, H. L.; Takami, M.; Thor, A. J. Quantities, Units and Symbols in Physical Chemistry, IUPAC Green Book, 3rd Ed., 2nd Printing, IUPAC \& RSC Publishing, Cambridge, 2008.

2. Vanhaecke, F.; Degryse, P. Isotopic Analysis: Fundamentals and Applications Using ICP-MS, WileyVCH Verlag \& Co. KGaA, Weinheim, Germany, 2012.

3. Balcaen, L.; Moens, L.; Vanhaecke, F. Spectrochim. Acta, Part B 2010, 65, 769.

4. Cheng, H.; Hu, Y. Environmental Pollution 2010, 158, 1134.

5. Díaz-Somoano, M.; Kylander, M. E.; López-Antón, M. A.; Suárez-Ruiz, I.; Martínez-Tarazona, M. R.; Ferrat, M.; Kober, B.; Weiss, D. J. Environ. Sci. Technol. 2009, 43, 1078.

6. Heumann, K. G.; Gallus, S. M.; Rädlinger, G.; Vogl, J. J. Anal. At. Spectrom. 1998, 13, 1001.

7. Albarède, F.; Beard, B. Reviews in Mineralogy \& Geochemistry 2004, 55, 113.

8. Yang, L. Mass Spectrom. Rev. 2009, 28, 990.

9. Weyer, S.; Schwieters, J. Int. J. Mass Spectrom. 2003, $226,355$.

10. Andersen, T.; Knutsen, A. B. Acta Chemica Scandinavica 1962, 16, 849 .

11. Strelow, F. W. E.; Toerien, F. S. Anal. Chem. 1966, 38, 545.

12. Guide to the Expression of Uncertainty in Measurement. International Organization for Standardisation (ISO), Geneva, 1995. 